\title{
Reseña de Antipode Editorial Collective (EDS.). (2019). Keywords in Radical Geography: Antipode at 50. Hoboken: Willey Blackwell.
}

\author{
David Herrera Santana \\ Facultad de Filosofía y Letras, Universidad Nacional Autónoma de México \\ México
}

En 2019, la revista Antipode: A Radical Journal of Geography cumplió 50 años de existencia y para celebrar una década más de vida se publicó un número especial, Keywords in Radical Geography: Antipode at 50. Fundada en la Clark University, Worcester, Massachusetts, en 1969, Antipode reivindicó una geografía radical (antisexista, anti-colonial, anti-racista, anti-clasista) en un contexto académico en donde estudiantes y profesores que demandaban lo radical estaban siendo expulsados o limitados por el mainstream disciplinar. "A partir de llamar 'una revista' a unas cuatro docenas de páginas, los fundadores de Antipode crearon una salida para la publicación de trabajos que eran extremadamente críticos con el status quo y dedicados a los ideales de la justicia social" (Castree et al., 2009: 3).

En sus inicios, Antipode adopta una crítica marxista que sustenta la radicalidad que reivindica. En la década de 1980, la revista abrió las puertas a otras perspectivas que permitieron, desde el punto de vista de quienes entonces engrosaban sus filas, una radicalidad y una crítica más amplia (feminismo, post-colonialismo, teorías postestructuralistas, teorizaciones "verdes") que se dirigía a delinear una nueva forma de conocimiento radical (Castree et al., 2009; Theodore et al., 2019). Sin embargo, en el número especial sobre el 40 aniversario, en 2009, Michael J. Watts realiza una autocrítica a tomar en consideración, a saber: que dicha radicalidad era muy utopista y que no contemplaba el contexto y los cambios en las condiciones sociales objetivas que estaban en marcha, por lo que no se despega del todo de un idealismo revolucionario.

Esa postura radical se transformó en una especie de ortodoxia que limitó mucho el propio alcance y sentido de la crítica. Son estos elementos los que sirven de pretexto para iniciar justamente el número especial Keywords in Radical Geography (Theodore et al., 2019), cuestionando la pertinencia y la vigencia del pensamiento radical y crítico en la geografía y el mundo de hoy. La introducción contiene la profunda introspección y autocrítica a la que se somete tanto la trayectoria como los contenidos de Antipode a lo largo de medio siglo de vida. La reflexión sobre la utilidad de la revista no solamente se dirige a contextualizarla en el momento actual, sino también a los 50 años de producción intelectual radical y crítica, que responde a diversos contextos históricos, etapas, preocupaciones y experiencias. 
Cabe recordar que el contexto de la fundación de Antipode es la revolución de 1968 (Wallerstein, 2010), las numerosas formas represivas de contención social, militarización, seguritización y contrainsurgencia en el mundo y las múltiples expresiones contestatarias frente a estas reacciones (Herrera y González, 2018). En este sentido, la fundación de Antipode trascendió la experiencia en el campo de geografía, la más relevante hasta entonces por la reivindicación que hace de la radicalidad. Se trata de una reflexión colectiva inscrita en el contexto de las múltiples rebeliones y subversiones epistemológicas que acontecieron en el pensamiento social frente al positivismo y el conservadurismo imperantes en la época y frente al utilitarismo característico de ciertos campos disciplinares, como el de la geografía, los estudios regionales y relaciones internacionales, entre otros vinculados entre sí en torno al conocimiento del mundo, la orientación problem-solving que configuran, así como la forma de plantear métodos y estrategias de intervención en diversas realidades y contextos sociales, con una mirada colonial-racista, patriarcal-heteronormativa y de clase. De ahí la advertencia que lanzara David Harvey en 1976 como una especie de mandato para las labores colectivas de Antipode:

Las condiciones sociales objetivas exigen que digamos algo sensato y coherente o bien para siempre (por falta de credibilidad o, peor aún, por un mayor deterioro de las condiciones sociales objetivas) permanecer en silencio. Son las emergentes condiciones sociales objetivas y nuestra incapacidad patente para hacer frente a ellas, lo que esencialmente explica la necesidad de una revolución en el pensamiento geográfico. (citado en Theodore et. al., 2019: 2)

Y Harvey no hablaba en el vacío ni sin un sentido práctico. Como ha sido analizado desde la propia crítica interna de quienes colaboran en Antipode (Castree et al., 2009; Theodore et al., 2019), en la década de 1970 la crisis de sobreacumulación se hace presente con gran fuerza y con reajustes espacio-temporales (Harvey, 2007) revolucionando la escala mundial - y el resto-; también fue el momento donde apareció un fuerte cuestionamiento de la propia utilidad de la crítica y del sentido de la revolución que se planteó en las páginas de Antipode. La prevalencia entonces de una ortodoxia marxista, junto con la abrumadora presencia de una academia anglosajona occidental, puso en cuestionamiento si se podían alcanzar objetivos radicales mientras se tenían visiones y pensamientos sesgados sobre la realidad (Castree et al., 2009; Theodore et al., 2019). Tanto la inclusión de otras miradas como la expansión hacia otras latitudes se transformó en una imperante necesidad estratégica (Theodore et al., 2019).

En el primer sentido, la apertura hacia otros enfoques y tradiciones teóricas conduce a la inclusión de numerosos referentes (Foucault, Negri, Latour, Said, Nancy, Rancière, Agamben, Haraway) que, al dialogar con aquellos referentes "tradicionales" (Marx, Gramsci, Polanyi), abrieron el campo de discusión, enriquecieron la mirada hacia políticas progresistas y formas radicales de pensamiento, en una línea que se aleja de la ortodoxia, sin recurrir al eclecticismo acrítico. Se afirmaba en el número especial sobre los 40 años: 


\section{$126 \square$ RESEÑAS}

Esta [crítica a partir de los nuevos referentes] a menudo toma la forma de enfoques más específicos y situados, en los que las políticas y prácticas ya existentes son reformuladas e interrogadas por su potencial transformador. Mientras que la economía, los Estados y los mercados tienden a aparecer como las categorías dominantes en los análisis político-económicos de la izquierda más convencional, los cuerpos espacializados, de género y racializados se hacen más visibles en estas miradas alternativas, como también lo hacen geográficamente procesos y prácticas específicas de imaginación y ensamblaje, así como la micropolítica de la emoción, el afecto y la ética. También hay una política de prefiguración marcada aquí ("sé el cambio que deseas ver" como decía Gandhi), que tiene como objetivo construir aspiraciones futuras alcanzables en el presente a través de una acumulación de pequeños cambios. Se trata de abrazar el "Poder juntos" en lugar del "poder sobre". (Castree et al., 2009: 5).

El cambio en perspectivas teórico-metodológicas se hizo acompañar de un intento de apertura hacia los conocimientos del "Sur" (Theory from de South) que se vio reflejado en el número editado en 1975 por Richard Peet y Milton Santos sobre "Subdesarrollo y dominación/dependencia", que no logró ser tan incluyente, ni tampoco tuvo el impacto que se pretendía en un inicio. No obstante, el llamado era a alejarse del anglo centrismo característico de las publicaciones (Theodore et al., 2019; Watts, 2009). A partir de entonces, Antipode ha albergado numerosas perspectivas y diversos análisis que se nutren de distintas tradiciones del pensamiento marxista, así como otras corrientes críticas cercanas, sin trascender el anglo centrismo y el occidentalismo, habiéndose centrado en una dinámica creciente en ciertos temas y autores (Theodore et al., 2019).

En ese sentido, la radicalidad de Antipode ha sido la crítica al capitalismo, a la mercantilización, al racismo, las formas heteronormativas y el clasismo como sustento de las relaciones desiguales y opresivas de poder que se configuran en diversas escalas (Theodore et al., 2019; Castree et al., 2009; Watts, 2009). Esto se debe a la intención constante de producir un conocimiento útil, uno que se dirija a producir la anhelada revolución que el neoliberalismo y el globalismo pop decretaron desterrada (Beck, 1998). Como expresó Neil Smith: "Una de las grandes violencias de la era neoliberal fue la clausura de la imaginación política" (2009: 56).

Es, quizá, con ello en mente, que para conmemorar sus 50 años Antipode haya pensado en las "palabras clave" (Keywords) para la geografía radical del siglo XXI, con la perspectiva histórica que da la reflexión de medio siglo; un siglo que, como afirmara Adolfo Gilly (2006), se caracteriza por una "casi increíble acumulación de violencia" (20). Las y los autores que contribuyen a esclarecer estas palabras clave se caracterizan no sólo por su trayectoria política e intelectual, sino también por su compromiso con la necesidad de un pensamiento centrado en la dimensión estratégica de la comprensión y la denuncia del mundo, con miras a su transformación.

De esta forma, encontramos dentro de la Keywords discusiones sobre la agnotología (el estudio de la ignorancia), el ethos democrático, la economía comunitaria, la contrahegemonía, las geografías decoloniales, la conciencia política, la globalización radical

y la discusión sobre lo común, que han sido temas recurrentes en el pensamiento social 
y en la geografía radical de las últimas cuatro décadas, junto con otras "palabras" que conducen la reflexión sobre las dimensiones y perspectivas críticas, ámbitos, contextos y preocupaciones actuales, que se conjugan con aquellas que habían ocupado el centro del pensamiento radical hasta ahora.

Cindi Katz, Lynda Johnston, Andrea Gibbons, David Wilson, Gavin Brown, Colin McFarlane, entre otros, además del equipo editor encargado de este número especial, son las mentes detrás del análisis puntual de las 50 Keywords contenidas en las 279 páginas de este número. Basándose en el título de la clásica obra de Raymond Williams, Keywords: A Vocabulary of Culture and Society, Antipode propone "palabras clave" que, en apariencia caóticas e inconexas, contribuyan a la reflexión colectiva, a exaltar la valía de 50 años de trabajo, a provocar nuevos análisis y a mostrar la necesidad de apertura y reflexión permanentes.

El significado de estas palabras es una pregunta abierta, y podría decirse que la tarea de explorar su significado y uso puede abrir nuevas posibilidades políticas, que de lo contrario podrían pasar desapercibidas. En palabras de Theodore:

Creemos que [los colaboradores] son un grupo persuasivo, apasionado y riguroso en sus reflexiones sobre por qué "Amor" y "Fragmentos", "Vulnerabilidad" y "Monumento", y toda clase de otras palabras extrañas y maravillosas hablan e importan en las historias de la geografía radical, su condición actual y las posibles direcciones futuras. Y en este sentido, cada entrada también podría ser el comienzo de una nueva conversación ... que es de lo que debe tratarse el proyecto radical. (Theodore et. al., 2019: 9).

Keywords in Radical Geography: Antipode at 50 es un valioso trabajo por la autocrítica que realiza, el recuento de la historia de Antipode y la introspección en su legado, la reflexión sobre la valía de un registro de pensamiento situado, comprometido con la transformación del mundo, así como por el aporte en torno a categorías y conceptos actuales para la geografía.

\section{Referencias}

BECK, Ulrich. (1998) ¿Qué es la globalización? Falacias del globalismo, respuestas a la globalización. Barcelona: Paidós.

Castree, Noel; Chatterton, Paul; Heynen, Nik; Larner, Wendy; y Wright, Melissa. (2009). "Introduction: The Point is to Change It". Antipode, 41 (1), 1-9.

Gilly, Adolfo. (2006). Historia a contrapelo. Una constelación. México: Era. Harvey, David. (2007). Espacios del capital. Madrid: Akal.

Herrera, David; y GonzÁlez, Fabián. (2018). "El espacio dominante y el legado post68 en México y el mundo: militarización, seguritización y violencia”. Revista Mexicana de Ciencias Políticas y Sociales, (234), 287-314.

Smith, Neil. (2009). "The Revolutionary Imperative”. Antipode, 41(1), 50-65. 


\section{$128 \square$ RESEÑAS}

Theodore, Nik; Jazeel, Tariq; Kent, Andy; y McKittrick, Katherine. (2019), “Keywords in Radical Geography: An Introduction". En Antipode Editorial Collective (Ed.). Keywords in Radical Geography: Antipode at 50. Hoboken: Wiley Blackwell. 2-13.

Wallerstein, Immanuel. (2010), Análisis de sistemas-mundo. Una introducción. México: Siglo XXI.

WatTs, Michael J. (2009). "Now and Then”. Antipode, 41(1), 10-26. 